\title{
Cervical human papillomavirus prevalence, risk factors and outcomes in a cohort of HIV-infected women in Harare, Zimbabwe
}

\begin{tabular}{|c|c|}
\hline \multicolumn{2}{|c|}{$\begin{array}{l}\text { Authors: } \\
\text { Ardele M. Mandiriri }{ }^{1,2} \text { (I) } \\
\text { Margaret J. Pascoe }^{1} \text { (I) } \\
\text { Tinei Shamu } \\
\text { Sara Lowe }{ }^{1,4} \text { (D) }\end{array}$} \\
\hline \multicolumn{2}{|c|}{$\begin{array}{l}\text { Affiliations: } \\
{ }^{1} \text { Newlands Clinic, Harare, } \\
\text { Zimbabwe }\end{array}$} \\
\hline \multicolumn{2}{|c|}{$\begin{array}{l}{ }^{2} \text { Department of Epidemiology, } \\
\text { Faculty of Epidemiology and } \\
\text { Population Health, London } \\
\text { School of Hygiene and } \\
\text { Tropical Medicine, London, } \\
\text { United Kingdom }\end{array}$} \\
\hline \multicolumn{2}{|c|}{$\begin{array}{l}{ }^{3} \text { Institute of Social and } \\
\text { Preventive Medicine, } \\
\text { University of Bern, Bern, } \\
\text { Switzerland }\end{array}$} \\
\hline \multicolumn{2}{|c|}{$\begin{array}{l}{ }^{4} \text { Department of Medicine, } \\
\text { College of Health Sciences, } \\
\text { University of Zimbabwe, } \\
\text { Harare, Zimbabwe }\end{array}$} \\
\hline \multicolumn{2}{|c|}{$\begin{array}{l}\text { Corresponding author: } \\
\text { Ardele Mandiriri, } \\
\text { ArdeleM@newlandsclinic. } \\
\text { org.zw }\end{array}$} \\
\hline \multicolumn{2}{|c|}{$\begin{array}{l}\text { Dates: } \\
\text { Received: } 06 \text { July } 2020 \\
\text { Accepted: } 30 \text { Aug. } 2020 \\
\text { Published: } 05 \text { Nov. } 2020\end{array}$} \\
\hline \multicolumn{2}{|c|}{$\begin{array}{l}\text { How to cite this article: } \\
\text { Mandiriri AM, Pascoe MJ., } \\
\text { Shamu T., Lowe S. Cervical } \\
\text { human papillomavirus } \\
\text { prevalence, risk factors and } \\
\text { outcomes in a cohort of } \\
\text { HIV-infected women in } \\
\text { Harare, Zimbabwe. S Afr J HIV } \\
\text { Med. 2020;21(1), a1123. } \\
\text { https://doi.org/10.4102/ } \\
\text { sajhivmed.v21i1.1123 }\end{array}$} \\
\hline \multicolumn{2}{|l|}{ Read online: } \\
\hline 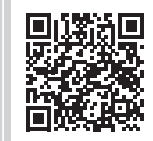 & $\begin{array}{l}\text { Scan this QR } \\
\text { code with your } \\
\text { smart phone or } \\
\text { mobile device } \\
\text { to read online. }\end{array}$ \\
\hline
\end{tabular}

Background: Human papillomavirus (HPV) associated invasive cervical cancer (ICC) is common in Zimbabwe, disproportionately affecting women living with HIV (WLHIV). Understanding high-risk HPV (hrHPV) infection in relation to cervical disease is important for ICC prevention amongst WLHIV.

Objectives: To describe the prevalence of cervical hrHPV, type distribution, associated risk factors and ICC screening outcomes in an urban cohort of Zimbabwean women.

Methods: In this cohort study, WLHIV were tested for hrHPV infection using the Cepheid Xpert ${ }^{\circledR}$ HPV assay and followed up for incident cervical disease. Follow-up assessments were done by visual inspection with acetic acid (VIA). Descriptive statistics and logistic regression were used to describe hrHPV burden and association between hrHPV and potential risk factors. Incidence rates (IR) and rate ratios of cervical disease by hrHPV infection status were also calculated.

Results: Amongst 321 WLHIV recruited, hrHPV prevalence was $24.9 \%(n=80)$. Fifty-two of these women $(65 \%)$ were positive for hrHPV types other than 16 or $18 / 45$. Younger age (22-29 years), early sexual debut (13-16 years) and antiretroviral therapy (ART) regimen (second-line ART) were independently associated with hrHPV positivity. Positive VIA IR ratio between hrHPV-positive and -negative women was 12.57 (95\% confidence interval [CI]: 4.14-38.19). Only women with hrHPV infection had incident cervical disease (IR: 6.41/100 person-years, (95\% CI: 3.33-12.32). There were no ICC cases by the end of the 2-year follow-up.

Conclusion: There was a high prevalence of hrHPV infection other than 16 and 18/45 in this cohort. Integrating HPV testing in cervical cancer screening programmes may increase screening intervals in hrHPV-negative women, reducing costs for programmes. We recommend further research into cross protectivity of the bivalent and quadrivalent HPV vaccines against these other hrHPV types.

Keywords: cervical cancer screening; HPV infection in WLHIV; hrHPV prevalence; visual inspection with acetic acid; HPV vaccine cross protection.

\section{Introduction}

Zimbabwe is one of the countries with the highest human immunodeficiency virus (HIV) burden in sub-Saharan Africa. In 2018, the HIV prevalence was estimated at $12.7 \%$ amongst adults aged 15 to 49 years, with a higher prevalence in women (15.4\%) than in men $(10.0 \%) .{ }^{1}$ Women living with HIV (WLHIV) have higher rates of infection with high-risk Human Papillomavirus (hrHPV) genotypes. High-risk human papillomavirus co-infection with HIV is associated with decreased clearance, increased HPV persistence and progression to precancerous lesions and cervical cancer. ${ }^{2,3,4,5}$ Invasive cervical cancer (ICC) is the most common female malignancy in Zimbabwe and is the leading cause of female cancer-related deaths with an age-standardised mortality rate of 46 per 100000 women per year. $^{6}$

The current recommended cervical cancer screening method in low resource settings such as subSaharan Africa is visual inspection of the cervix after application of acetic acid (VIA). ${ }^{7,8}$ The WHO 2013 cervical cancer guidelines recommend primary HPV testing as the preferred method where resources are available, as HPV testing is more sensitive and effective in identifying women at

Copyright: @ 2020. The Authors. Licensee: AOSIS. This work is licensed under the Creative Commons Attribution License. 
high risk for precancerous and cancerous lesions. ${ }^{10,11}$ This modality of screening has not been rolled out in the region because of the prohibitive cost of the test. The diagnostic landscape is changing, and as cost-effective point-of-care HPV DNA tests are becoming more accessible, countries have commenced HPV screening as the preferred primary cervical cancer screening method. ${ }^{12,13}$

The Zimbabwean government partnering with GAVI, the Vaccine Alliance in 2018, rolled out a national HPV vaccination programme, as its cervical cancer primary prevention method. The programme targeted the countries' 800000 girls aged 10-14 using the bivalent vaccine, which confers protection to hrHPV subtypes $16 / 18 / 45 .{ }^{14}$ This, however, has raised questions on the cross protective effect of other hrHPV subtypes, which have been reported to be prevalent amongst women in Zimbabwe. ${ }^{15,16}$

Sub-Saharan African studies investigating HPV prevalence in WLHIV have shown a wide variability across different countries from $24 \%$ to $64 \%$. $17,18,19,20,21,22$ In 2017, a Zimbabwean study showed a hrHPV prevalence of $33 \%$ in a rural cohort of 123 WLHIV. Types 35, 52 and 58 were found to be amongst the highly prevalent types, in addition to types 16 and $18 .{ }^{15}$ These geographical differences in HPV burden and type distribution highlight the need to assess the Zimbabwean urban context independently. In this cohort study, we investigated the prevalence of hrHPV, the associated risk factors and VIA outcomes during 2 years of follow-up.

\section{Methods \\ Study design and setting}

This analytic cohort study was conducted amongst WLHIV at Newlands Clinic (NC), in Harare, Zimbabwe. The NC was established in 2004 in partnership with the Ministry of Health and Child Care (MoHCC) and provides comprehensive HIV care to approximately 6500 men, women and children. ${ }^{23}$ Sexual reproductive health services form an integral component of HIV care at NC and include cervical cancer screening, diagnosis and treatment of sexually transmitted infections (STIs), and the provision of short- and long-acting reversible contraceptive methods. An HPV vaccination programme was also introduced at the clinic in 2015. In this year alone, 517 adolescents and youth 12-22 years old (312 females and 205 males) were vaccinated using the quadrivalent HPV vaccine. The vaccination of children, adolescents and young adults still continues to date at the clinic as part of the clinic's effort to prevent a potential burden of HPV-related cancers in these young people as they mature.

Women registered in care at NC attend routine annual cervical cancer screening where the VIA method is used as guided by the Zimbabwean Ministry of Health. The procedure is performed by a team of three nurses who have been trained in this technique. Women with a positive VIA screen are treated with either cryotherapy, loop electrical excision procedure (LEEP) or referred for specialist management depending on the type and extent of the cervical lesion. Visual inspection with acetic acid and cryotherapy are conducted by the nurses. The LEEP procedure is performed by a gynaecologist, and the excised tissue is sent to an external laboratory for histological examination.

\section{Study procedure}

We invited women attending cervical cancer screening between 01 September 2017 and 21 November 2018 to participate in the study. Sexually active, HIV-positive women above the age of 18 years who consented to participate in the study were recruited. Potential participants with a positive pregnancy test were excluded. Baseline information including sexual history and demographic information were collected and stored in a Microsoft Access database.

At the baseline visit, we screened participants using VIA, the study nurses collected endocervical swabs for the HPV DNA tests. Women who screened positive by VIA were reviewed six monthly, and those who screened negative, annually. For the HPV DNA tests, we collected endocervical swabs using the Cervexbrush25 and deposited the samples into Preservcyt50 transport medium. We used Xpert ${ }^{\circledR}$ HPV kits for testing on the GeneXpert machine located at the onsite NC laboratory. The test detected 14 high-risk HPV with callouts for HPV 16, HPV 18/45 and 'other hrHPV'. Amongst the 'other hrHPV' subtypes are HPV 31, 33, 35, 39, 51, 52, 56, 58, 59, 66 and 68. The $14 \mathrm{HPV}$ types are detected in five fluorescent channels, each with individual parameters for target detection and validity; channel 1: HPV16, channel 2: HPV18/45, channel 3: HPV31/33/35/52/58, channel 4: HPV51/59, channel 5: HPV 39/56/66/68. For channels in which more than one type is detected, the Xpert ${ }^{\circledR}$ HPV test does not distinguish between types. ${ }^{24}$ Positive hrHPV results during the study period did not alter VIA follow-up intervals which were determined by VIA results only. We followed up participants with VIA screening for a median of 26 months (interquartile range [IQR]: 24-28), which was up to April 2020. Participants with incident HPV-related disease as highgrade vulva intraepithelial neoplasia (VIN3) were censored from further follow-up.

We collected additional patient data including antiretroviral therapy (ART) medication history, VIA screening results and laboratory results from the clinic's electronic database. None of the women in the study cohort had an HPV vaccination history.

\section{Statistical analysis}

We used Stata 13.1 (College Station, Texas) for data cleaning and analysis. We used proportions and medians for descriptive statistics, logistic regression and the associated Wald test for measures of association between hrHPV test and potential risk factors, and Wilcoxon Rank-Sum (Mann Whitney) test differences between continuous 
variables. We assessed all potential risk factors in univariate and multivariable logistic regression models to determine independent associations. Our final logistic regression model did not include VIA data as HPV infection is known to precede VIA status. Incidence rates (IR) and IR ratios for cervical disease defined as VIA positivity, high-grade squamous intraepithelial neoplasia (HGSIL) and cervical cancer were assessed with the follow-up data. All statistical tests were two-tailed with $p<0.05$ considered significant and $95 \%$ confidence intervals [CI] used. In summary, our descriptive, univariate and multivariate analysis were based on all data $100 \%(n=321)$. Follow-up data was based on $96 \%$ ( $n=308)$ women with hrHPV test results and without incident cervical disease.

\section{Ethical consideration}

The study was approved by the Newlands Clinic Research team and the Medical Research Council of Zimbabwe (Approval MRCZ/A/1980).

\section{Results}

We recruited 321 women with a median age of 44 years (IQR: $38-50)$. One hundred and thirty-eight (43.0\%) women were married and $29.6 \%(n=95)$ were widowed. Two-thirds of the women $(63.9 \%, n=205)$ were educated to high-school level (at least 8 years of formal education). Most women (64.5\%, $n=207$ ) reported sexual debut between 17 and 21 years, and $13.4 \%(n=43)$ were 16 years or below at sexual debut. Regarding duration on ART, 233 (70.8\%) had been on ART for more than 5 years (median ART duration $=7.8$ years, IQR: 4.4-10.8 years). In total, 252 (78.5\%) were on a first-line ART regimen (1 Non-nucleoside Reverse Transcriptase Inhibitor [NNRTI] + 2 Nucleoside/Nucleotide Reverse Transcriptase Inhibitor [NRTI]) and had a median CD4 count of 525 cells / $\mathrm{mm}^{3}$ (IQR 366-687 cells $/ \mathrm{mm}^{3}$. One-third $(33.7 \%, n=108)$ reported that they were unaware of their partner's HIV status and $15.6 \%(n=50)$ were in HIV sero-discordant relationships. Amongst the 310 women who had HIV viral load results, 91.2\% $(n=283)$ had undetectable viral loads (below 50 copies $/ \mathrm{mL})$ and $2.9 \%(n=9)$ had a viral load above 1000 copies/mL (Table 1).

All the 321 women had a successful HPV test, 80 (24.9\%; 95\% CI: $20.2 \%-29.7 \%$ ) were positive for any hrHPV. Human papillomavirus 16 positivity without other coinfections was in $9(11.2 \%)$ women, 10 (12.5\%) were positive for HPV 18/45 alone, 52 (65\%) for other hrHPV. A small proportion, four (5\%) had HPV 16 and other hrHPV coinfections, and five (6.3\%) had HPV 18 and other hrHPV. There were no co-infections with HPV 16 and HPV 18/45. Data for specific channels positive for other hrHPV-positive samples were available for 56/61 samples (91.8\%). Amongst these 56 samples, 40 (71.4\%) were positive in channel 3 (HPV31/33/35/52/58), 5 (7.1\%) in channel 4 (HPV51/59) and $15(26.8 \%)$ in channel 5 (HPV $39 / 56 / 66 / 68)$. One was positive in both channels 3 and 4 , whilst two were positive in both channels 3 and 5 (Figure 1).
Of the 80 women with a positive hrHPV test, $6(7.5 \%)$ had a concurrent VIA positive result. Of these women, two were positive for HPV type 16 only, one for HPV 16 and other types (channel 5) and three tested positive for other hrHPV types ( 2 were channel 3 positive and the other had missing channel data).

In the univariate analysis shown in Table 2, women aged 22-29 years (OR 3.0, 95\% CI: 1.2-7.9, $p=0.02$ ), women with a positive VIA within 2 years prior to HPV screening (OR 6.1, 95\% CI: 2.9-12.8, $p<0.001$ ), women on second-line ART (OR 2.38, 95\% CI: 1.3-4.4) and those with a positive VIA at time of HPV screen (OR 6.6, 95\% CI: 1.6-27.6, $p<0.001)$ were more likely to test positive for hrHPV. Having a detectable viral load at the time of screening and sexual debut between the age of 13 and 16 years were also significantly associated with hrHPV positivity (OR: 2.3, 95\% CI: $1.01-5.3, p=0.04$, OR:3.2, CI: 1.3-7.9, $p<0.01)$. However, no statistical significance for hrHPV positivity was observed for CD4 count $<200$ cells $/ \mathrm{mm}^{3}$ (OR 1.2, CI: 0.4-4.0). In the multivariate analysis, only younger age (22-29 years), (adjusted odds ratio [aOR] 2.9; 95\% CI: $1.0-8.8, p=0.05$ ), ART regimen (aOR 2.1; 95\% CI: 1.1-4.0, $p<0.05$ ) and early sexual debut at ages 13-16 years (aOR 4.0, 95\% CI: 1.4-11.5, $p<0.05)$ showed evidence of association with hrHPV positivity (Table 2).

\section{Visual inspection with acetic acid follow-up outcomes after high-risk human papillomavirus testing}

Three hundred and eight (96\%) participants with negative VIA at the time of hrHPV screen were followed up for a median of 2 years (645 total person-years of follow-up). Follow-up was made to determine incident VIA positivity, incident HGSIL and incident cervical cancer (confirmed by LEEP biopsy) by HPV infection status at baseline. The VIA positivity IR in hrHPV-negative women was 0.79 per 100 person-years (95\% CI: 0.30-2.11). In hrHPV-positive women, the VIA positivity IR was 9.97 per 100 person-years (95\% CI: 5.90-16.83). Incident rate ratio between the two groups of women was 12.57 (95\% CI: 4.14-38.19). There was no evidence of incident cervical disease (HGSIL) in women who tested negative for hrHPV infection. Amongst women with hrHPV infection, the IR of HGSIL was 6.41 per 100 person-years (95\% CI: 3.33-12.32). During the follow-up period, there was no incident of cervical cancer regardless of hrHPV infection status (Figure 2).

Of the 14 incident VIA-positive women at follow-up, eight $(57 \%)$ at baseline were positive for other hrHPV subtypes (four being channel 3 subtypes and the others having missing channel data), two were positive for HPV16, one was positive for HPV 16 and other hrHPV subtypes (channel 5). Two were positive for hrHPV 18/45 only and one for hrHPV 18/45 and other (channel 3). None of the women had $16 / 18 / 45$ coinfections at baseline. None of the women with positive channel 4 subtypes had incident cervical disease at follow-up. 
TABLE 1: Socio-demographic characteristics and risk factors of participants at baseline $(N=321)$.

\begin{tabular}{|c|c|c|c|c|c|c|}
\hline \multirow[t]{3}{*}{ Characteristic } & \multicolumn{6}{|c|}{ Frequency $(\%) \dagger$} \\
\hline & \multicolumn{2}{|c|}{ hrHPV negative $N=241$} & \multicolumn{2}{|c|}{ hrHPV positive $N=80$} & \multicolumn{2}{|c|}{ Overall $N=321$} \\
\hline & $n$ & $\%$ & $n$ & $\%$ & $n$ & $\%$ \\
\hline Age (years), median (IQR) & 44 & $39-50$ & 42 & $37-49$ & 44 & $38-50$ \\
\hline \multicolumn{7}{|l|}{ Age bands (years) } \\
\hline $22-29$ & 11 & 4.6 & 10 & 12.5 & 21 & 6.5 \\
\hline $30-45$ & 121 & 50.2 & 36 & 45.0 & 157 & 48.9 \\
\hline $46-60$ & 89 & 36.9 & 32 & 40.0 & 121 & 37.7 \\
\hline$>60$ & 20 & 8.3 & 2 & 2.5 & 22 & 6.9 \\
\hline \multicolumn{7}{|l|}{ Marital status } \\
\hline Divorced & 23 & 9.5 & 14 & 17.5 & 37 & 11.5 \\
\hline Married & 107 & 44.4 & 31 & 38.8 & 138 & 43.0 \\
\hline Separated & 10 & 4.1 & 3 & 3.8 & 13 & 4.1 \\
\hline Single & 31 & 12.9 & 7 & 8.8 & 38 & 11.8 \\
\hline Widowed & 70 & 29.1 & 25 & 31.3 & 95 & 29.6 \\
\hline \multicolumn{7}{|l|}{ Gravidity } \\
\hline $1-3$ & 144 & 59.8 & 47 & 58.8 & 191 & 59.5 \\
\hline$>3$ & 90 & 37.3 & 29 & 36.3 & 119 & 37.1 \\
\hline \multicolumn{7}{|l|}{ Contraception } \\
\hline Hormonal & 64 & 26.6 & 29 & 36.3 & 93 & 29.0 \\
\hline Non-hormonal & 47 & 19.5 & 20 & 25.0 & 67 & 20.9 \\
\hline None & 130 & 53.9 & 31 & 38.8 & 161 & 50.2 \\
\hline \multicolumn{7}{|l|}{ Education } \\
\hline None & 12 & 5.0 & 6 & 7.5 & 18 & 5.6 \\
\hline Primary school & 39 & 16.2 & 13 & 16.3 & 52 & 16.2 \\
\hline High school & 153 & 63.5 & 52 & 65.0 & 205 & 63.9 \\
\hline Tertiary education & 37 & 15.4 & 9 & 11.3 & 46 & 14.3 \\
\hline \multicolumn{7}{|l|}{ Age of sexual debut } \\
\hline$<13$ & 3 & 1.2 & 2 & 15.0 & 5 & 1.6 \\
\hline $13-16$ & 23 & 9.5 & 15 & 63.8 & 38 & 11.8 \\
\hline $17-21$ & 156 & 64.7 & 51 & 18.8 & 207 & 64.5 \\
\hline Negative & 40 & 16.6 & 10 & 12.5 & 50 & 15.6 \\
\hline Positive & 123 & 51.0 & 40 & 50.0 & 163 & 50.8 \\
\hline Unknown & 78 & 32.4 & 30 & 37.5 & 108 & 33.6 \\
\hline \multicolumn{7}{|l|}{ VIA + : At HPV screening } \\
\hline Positive & 3 & 1.2 & 6 & 7.5 & 9 & 2.8 \\
\hline Negative & 237 & 98.3 & 74 & 92.5 & 311 & 96.9 \\
\hline Missing & 1 & 0.4 & - & - & 1 & 0.3 \\
\hline \multicolumn{7}{|c|}{ Historical VIA outcomes 2 years prior to HPV sample } \\
\hline All negative & 226 & 93.8 & 57 & 28.8 & 283 & 11.8 \\
\hline Any positive & 15 & 6.2 & 23 & 71.3 & 38 & 88.1 \\
\hline Historical STI's 2 years prior to HPV san & & & & & & \\
\hline Any STI & 202 & 16.2 & 59 & 26.2 & 261 & 18.7 \\
\hline Non-Viral STI & 39 & 83.8 & 21 & 73.8 & 60 & 81.3 \\
\hline Duration of ART (years), median (IQR) & 7.6 & $4.6-10.5$ & 8.0 & $3.5-11.0$ & 7.8 & $4.4-10.8$ \\
\hline Viral load status§ (copies/mL) & & & & & & \\
\hline$<50$ & 218 & 90.5 & 65 & 81.3 & 283 & 3.4 \\
\hline 50-199 & 10 & 4.2 & 5 & 6.3 & 15 & 2.8 \\
\hline 200-1000 & 0 & 0.0 & 3 & 3.8 & 3 & 0.9 \\
\hline$>1000$ & 6 & 2.5 & 3 & 3.8 & 9 & 4.8 \\
\hline Missing & 7 & 2.9 & 4 & 5.0 & 11 & 88.2 \\
\hline CD4 count (cells $/ \mathrm{mm}^{3}$ ) & & & & & & \\
\hline Median (IQR) & 515 & $360-685$ & 481 & $354-622$ & 513 & $354-677$ \\
\hline$<200$ & 10 & 4.2 & 4 & 5.0 & 14 & 4.4 \\
\hline$\geq 200$ & 231 & 95.9 & 76 & 95.0 & 307 & 95.6 \\
\hline ART regimen & & & & & & \\
\hline ART naïve & 4 & 1.7 & 0 & 0.0 & 4 & 1.3 \\
\hline First line & 200 & 83.0 & 56 & 70.0 & 256 & 81.0 \\
\hline Second line & 36 & 14.9 & 24 & 30.0 & 60 & 18.7 \\
\hline Third line & 1 & 0.4 & 0 & 0.0 & 1 & 0.3 \\
\hline
\end{tabular}

VIA, visual inspection with acetic acid; ART, antiretroviral therapy; IQR, interquartile range; hrHPV, high-risk HPV; HPV, Human papillomavirus; STI, sexually transmitted infection.

$\dagger$, Frequency unless otherwise stated.

$\$$, One patient had cytology instead of VIA at HPV screen.

$\S$, Eleven patients did not have viral load. 


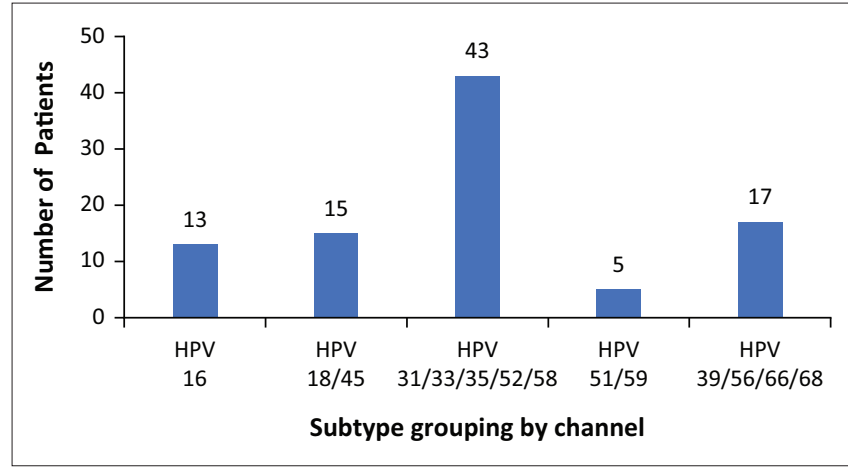

HPV, human papillomavirus.

FIGURE 1: Distribution of high-risk human papillomavirus infection in women living with human immunodeficiency virus $(n=80)$ as determined by five channel Xpert" human papillomavirus.

\section{Discussion}

We detected hrHPV infection in $24.5 \%$ of WLHIV attending routine cervical screening. The prevalence of types 16 and $18 / 45$ was relatively low, with a predominance $(65 \%)$ of the non-16/18/45 HPV subtypes. Our observed overall hrHPV prevalence is similar to the $33 \%$ prevalence reported in a cohort of WLHIV in rural Zimbabwe, ${ }^{15}$ but lower than that observed in other regional studies from Malawi, Burundi, Kenya and South Africa, which range from $45 \%$ to $64 \% .{ }^{19,20,25,26}$ The difference in reported prevalence rates between studies may be because of several factors including the heterogeneity of the study populations. Our study describes prevalence in an urban cohort of women with well-controlled HIV (95\% VL $<1000$ copies $/ \mathrm{mL}$ ). The majority of the women were receiving their first-line ART regimen for a median of 7 years and had previous cervical cancer screening.

Globally, HPV types 16 and 18 predominate and are responsible for most anogenital HPV-related cancers in women. ${ }^{27}$ In our study, 35\% of women with hrHPV had either HPV 16 or $18 / 45$, whilst $65 \%$ had 'other' high-risk types. These findings are consistent with data from other studies in the region, including a world regional HPV survey, which describes an increased probability amongst WLHIV to harbour other hrHPV types such as 31, 33, 35, 52 and 58, often in the absence of cervical disease (HGSIL or ICC). ${ }^{22,28,29,30}$. Cumulative risk and annual rate of progression to cancer varies depending on HPV type in immunocompetent women. ${ }^{31}$ In a longitudinal study of 11573 HIV-negative, hrHPV-positive women, Demarco et al. reported that $21.5 \%$ of women at Kaiser Permanente in Northern California, United States of America, with baseline HPV 16 had progressed to precancer/cancer at 7 years and women with HPV 33 also showing high cumulative risk with 18.4\% progression at 7 years. ${ }^{31}$ Our study's limited follow-up time inhibits us from making comments on the progression potential of the observed non-16/18/45 hrHPV subtypes. WLHIV with hrHPV are, however, at an increased risk of rapid progression to cervical disease. ${ }^{2,3,4,5}$ More robust epidemiological data in WLHIV are required to clearly define this risk according to hrHPV type with longer follow-up
TABLE 2: Univariate and multivariate analysis of patient characteristics associated with high-risk human papillomavirus positivity.

\begin{tabular}{|c|c|c|c|c|}
\hline Characteristic & OR $(95 \% \mathrm{Cl})$ & $p^{* *}$ & aOR $(95 \% \mathrm{Cl})$ & $p^{* *}$ \\
\hline \multicolumn{5}{|l|}{ Age bands (years) } \\
\hline $22-29$ & $3.0(1.2-7.9)$ & 0.02 & $2.9(1.0-8.8)$ & 0.05 \\
\hline $30-45$ & - & - & - & - \\
\hline $46-60$ & $1.2(0.7-2.1)$ & 0.50 & $1.6(0.8-3.2)$ & 0.17 \\
\hline$>60$ & $0.3(0.1-1.5)$ & 0.15 & $0.3(0.1-1.7)$ & 0.18 \\
\hline \multicolumn{5}{|l|}{ Marital status } \\
\hline Married & $0.8(0.5-1.3)$ & 0.38 & $0.8(0.4-1.5)$ & 0.49 \\
\hline Single & - & - & - & - \\
\hline \multicolumn{5}{|l|}{ Contraception } \\
\hline Non-hormonal & - & - & - & - \\
\hline Hormonal & $1.6(0.9-2.7)$ & 0.10 & $1.3(0.7-2.6)$ & 0.42 \\
\hline \multicolumn{5}{|l|}{ Gravidity } \\
\hline 0 & - & - & - & - \\
\hline $1-3$ & $0.6(0.2-2.0)$ & 0.39 & $0.7(0.1-3.2)$ & 0.62 \\
\hline$>3$ & $0.6(0.2-2.1)$ & 0.39 & $0.7(0.1-3.7)$ & 0.67 \\
\hline \multicolumn{5}{|l|}{ Education } \\
\hline None & $2.1(0.6-7.0)$ & 0.25 & $2.1(0.5-8.4)$ & 0.29 \\
\hline Primary school & $1.4(0.5-3.6)$ & 0.52 & $0.8(0.3-2.4)$ & 0.66 \\
\hline High school & $1.4(0.6-3.1)$ & 0.41 & $1.0(0.4-2.3)$ & 0.98 \\
\hline Tertiary & - & - & - & - \\
\hline \multicolumn{5}{|c|}{ Age of sexual debut } \\
\hline$<13$ & $3.3(0.5-21.8)$ & 0.22 & $4.6(0.6-33.8)$ & 0.13 \\
\hline $13-16$ & $3.2(1.3-7.9)$ & $0.01 * *$ & $4.0(1.4-11.5)$ & $0.01^{* *}$ \\
\hline $17-21$ & $1.6(0.8-3.2)$ & 0.18 & $1.4(0.6-3.0)$ & 0.41 \\
\hline$>21$ & - & - & - & - \\
\hline \multicolumn{5}{|c|}{ VIA at HPV sample collection } \\
\hline Positive & $6.6(1.6-27.6)$ & 0.001 & - & - \\
\hline Negative & - & - & - & - \\
\hline \multicolumn{5}{|c|}{ Viral load (copies/mL) } \\
\hline$<50$ & - & - & - & - \\
\hline$\geq 50$ & $2.3(1.0-5.3)$ & 0.04 & $1.6(0.6-4.2)$ & 0.34 \\
\hline \multicolumn{5}{|c|}{ STI history in the previous $\mathbf{2}$ years } \\
\hline Any STI & - & - & - & - \\
\hline Non-viral STI & $2.0(1.0-3.4)$ & 0.05 & $1.95(1.0-3.9)$ & 0.06 \\
\hline \multicolumn{5}{|c|}{ CD4 count (cells/mm³) } \\
\hline$<200$ & $1.2(0.4-4.0)$ & 0.75 & $0.82(0.2-3.2)$ & 0.77 \\
\hline$\geq 200$ & - & - & - & - \\
\hline \multicolumn{5}{|c|}{ VIA positive history in previous 2 years } \\
\hline Negative & - & - & - & - \\
\hline Positive & $6.1(2.9-12.8)$ & $<0.001$ & - & - \\
\hline \multicolumn{5}{|l|}{ ART regimen } \\
\hline First-line ART & - & - & - & - \\
\hline Second-line ART & $2.38(1.3-4.4)$ & $0.003^{* *}$ & $2.1(1.1-4.0)$ & $0.03^{* *}$ \\
\hline
\end{tabular}

VIA, visual inspection with acetic acid; ART, antiretroviral therapy; hrHPV, high risk HPV; HPV, Human papillomavirus; STI, sexually transmitted infection; $\mathrm{Cl}$, confidence interval; OR, odds ratio; $\mathrm{aOR}$, adjusted odds ratio.

**, Wald test $p$-values for variables associated with HPV infection in the univariate and bivariate analysis.

periods, in order to inform and optimise cervical screening programmes in WLHIV.

Human papillomavirus vaccination programmes are a key component of primary prevention to reduce cervical cancerassociated morbidity and mortality. The WHO currently recommends both bivalent and quadrivalent vaccines, which confer protection against hrHPV types 16 and $18 .^{32}$ Cross protection from the bivalent vaccine for other non-vaccine high-risk types as in our cohort has been demonstrated. Van De Weele et al. noted significant reductions in incident and/ or persistence of certain types (HPV 31, 33, 35 and 45), 


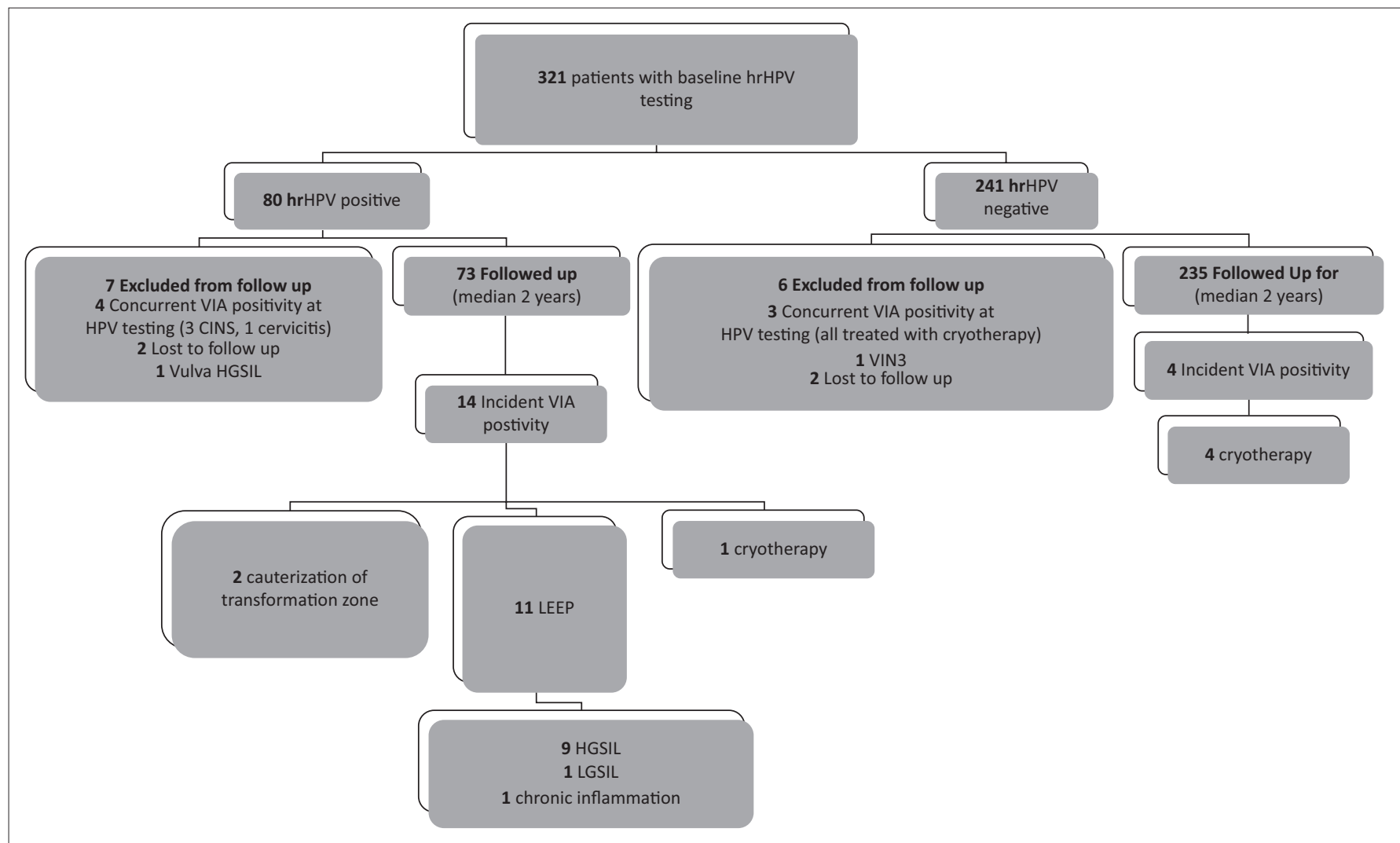

HPV, human papillomavirus; hrHPV, high-risk HPV; VIA, visual inspection with acetic acid; LEEP, loop electrical excision procedure; HGSIL, high grade squamous intraepithelial neoplasia; LGSIL, low grade squamous intraepithelial neoplasia; VIN3, vulva intraepithelial neoplasia; CIN3, cervical intraepithelial neioplasia 3.

FIGURE 2: Visual inspection with acetic acid follow-up outcomes after baseline high-risk human papillomavirus testing.

following exposure to the bivalent vaccine in a cohort of young Dutch women. ${ }^{33}$ In view of the dominance of subtypes other than HPV 16 or HPV 18/45, it is unclear whether the bivalent vaccine currently being administered in the Zimbabwe national programme will provide cross protection against these subtypes. An understanding of the degree of cross protection from context-specific data is warranted. This can be achieved through country-specific vaccine evaluation studies in the vaccinated cohort.

Early sexual debut and younger age were significant predictors of hrHPV infection in our cohort. Women with an early sexual debut (13-16 years) were at least three times more likely to test positive for hrHPV when compared with those whose sexual debut was after 21 years. In keeping with these findings, a cohort analysis of 1445 urban women in South Africa showed a peak in hrHPV prevalence in women younger than 25 years and a gradual decrease of hrHPV infection with increasing age of women. ${ }^{34}$ Van Aardt et al. postulate that the higher hrHPV prevalence in younger women may be partially explained by the lack of natural immunity in the initial stages of sexual activity. ${ }^{35}$ Literature also provides evidence that over $35 \%$ of women contract HPV within the first 2 years of sexual debut ${ }^{8}$ and that early sexual debut is also associated with a greater number of sexual partners and an increased risk of STI infection. ${ }^{36,37}$ Research to further elucidate clearance and progression to cervical dysplasia in younger women is also necessary.
Despite most women in our study being on first-line ART and $95 \%$ having Viral load (VL) < 1000 copies $/ \mathrm{mL}$, women on second-line therapy were twice as likely to test positive for hrHPV. In a systematic review of the association between HPV infection and ART, Menon et al. indicate that severe immuno-suppression of CD 4 counts $<200$ cells $/ \mathrm{mm}^{3}$ increases the risk of hrHPV infection. ${ }^{38}$ In our context, women on secondline ART often have a history of severe immunosuppression necessitating the switch to second-line ART.

After a median of 2 years follow-up, the incident rates for both VIA positivity and HGSIL were significantly higher in the women with hrHPV infection. No cases of HGSIL were observed in hrHPV-negative women. These data are consistent with findings from large longitudinal studies. Data from the Kaiser Portland cohort of over 20000 women and more than 15 years of follow-up demonstrate a low risk of precancer or cancer in HIV-negative women with a single negative hrHPV result. The high negative predictive value of HPV DNA testing is now well established, hence the adoption by many global cervical screening programmes as the primary screening tool. ${ }^{10,39}$ In low-income countries, lessfrequent cervical cancer screening, once every 3 years, for hrHPV-negative WLHIV will have both the potential to reduce costs and divert scarce resources to hrHPV-positive women who are at higher risk of developing cervical cancer and in women who have never been screened. This resource optimisation may translate to greater screening potential in 
settings where coverage is still low like in Zimbabwe. ${ }^{8}$ However, a key component of the potential success of this screening programme that utilises HPV testing is the development and availability of low-cost HPV assays.

As reported in the methodology, the use of the $\mathrm{Xpert}^{\circledR} \mathrm{HPV}$ kits limited our ability to describe individual hrHPV types in the cohort apart from HPV 16. Secondly, the duration of follow-up was short and because of the nature of the pathogenesis of HPV infection, there was limited time for disease progression to occur. Thirdly, hrHPV infection was only assessed at baseline, therefore questions of HPV persistence and clearance could not be addressed. Despite these limitations, the information from this study can be used to provide background data for prospective cohort studies to ascertain persistence of HPV infections in women with controlled HIV infection.

\section{Conclusion}

Our study highlights key epidemiological information on hrHPV infection in an urban cohort of HIV-infected women in Zimbabwe. There was a lower than expected overall prevalence of hrHPV infection in the study cohort, but a higher prevalence of hrHPV subtypes other than HPV 16 or $18 / 45$. This raises some important vaccine effectiveness questions regarding the current roll-out of the prequalified bivalent and quadrivalent HPV vaccine, particularly regarding the level of cross-protection against other hrHPV types, which are prevalent in our setting. Our follow-up data highlights the potential benefits of integrating primary HPV testing, in cervical cancer screening programmes amongst WLHIV, which may lead to reduced screening intervals in hrHPV-negative women and potentially result in cost reduction.

\section{Acknowledgements}

The authors are grateful to Sr Petronella Mudhokwani, Sr Grace Dambanemweya and Sr Adelaide Mawora, for their work in data collection. The authors are also grateful to Prof. Ruedi Luethy, for his support and guidance in this work.

\section{Competing interests}

The authors declare that they have no financial or personal relationships that may have inappropriately influenced them in writing this article.

\section{Authors' contributions}

A.M.M. was responsible for drafting the manuscript, managing the study data and follow-up of patients. M.J.P. was responsible for study conception, supervising the research process and contributed to the analysis of the data and write-up. S.L. was responsible for the conception of the study and supervising the drafting of the manuscript and analysis of data. T.S. conducted the data analysis and results write-up and assisted with study conception. All the authors revised the draft critically and gave final approval of the version to be published. All authors read and approved the final manuscript.

\section{Funding information}

The study was financed through the Newlands Clinic operational budget.

\section{Data availability statement}

Data are available upon request from the corresponding author.

\section{Disclaimer}

The views expressed in this article are the authors' and not necessarily reflect the official policy or position of any affiliated agency of the authors.

\section{References}

1. Zimbabwe | UNAIDS [homepage on the Internet]. [cited 2020 May 28]. Available from: https://www.unaids.org/en/regionscountries/countries/zimbabwe

2. Liu G, Sharma M, Tan N, Barnabas RV. HIV-positive women have higher risk of human papilloma virus infection, precancerous lesions, and cervical cancer. AIDS 2018 Mar 27;32(6):795-808. https://doi.org/10.1097/qad.0000000000001765

3. Looker KJ, Rönn MM, Brock PM, et al. Evidence of synergistic relationships between HIV and human papillomavirus (HPV): Systematic reviews and metaanalyses of longitudinal studies of HPV acquisition and clearance by HIV status, and of HIV acquisition by HPV status. J Int AIDS Soc. 2018;21(6):e25110. https:// doi.org/10.1002/jia2.25110

4. Mukanyangezi M, Rugwizangoga $B$, Manzi $O$, et al. Persistence rate of cervical human papillomavirus infections and abnormal cytology in Rwanda. HIV Med [serial online]. 2019 Aug [cited 2019 Nov 28];20(7):485-495. Available from: https://doi.org/10.1111/hiv.12782

5. Mudini W, Palefsky JM, Hale MJ, et al. Human papillomavirus genotypes in invasive cervical carcinoma in HIV-seropositive and HIV-seronegative women in Zimbabwe. J Acquir Immune Defic Syndr. 2018 Sep 01;79(1):e1-e6. https://doi. Zimbabwe. J Acquir Immune Defic Synd
org/10.1097/qai.0000000000001754

6. Human papillomavirus and related diseases report ZIMBABWE [document on the Internet]. [cited 2020 Aug 03]. Available from: www.hpvcentre.net

7. Msyamboza KP, Phiri T, Sichali W, Kwenda W, Kachale F. Cervical cancer screening uptake and challenges in Malawi from 2011 to 2015: Retrospective cohort study. BMC Public Health. 2016 Aug 17;16(1):806. https://doi.org/10.1186/s12889-0163530-y

8. Kuguyo O, Matimba A, Tsikai N, et al. Cervical cancer in Zimbabwe: A situation analysis. Pan Afr Med J. 2017;27:215. https://doi.org/10.11604/pamj.2017.27.215.12994

9. Parham GP, Mwanahamuntu MH, Kapambwe S, et al. Population-level scale-up of cervical cancer prevention services in a low-resource setting: Development, implementation, and evaluation of the cervical cancer prevention program in Zambia. PLoS One [serial online]. 2015 Apr 17 [cited 2020 Mar 06];10(4):e0122169. Available from: https://doi.org/10.1371/journal.pone.0122169

10. WHO guidelines. WHO guidelines for screening and treatment of precancerous lesions for cervical cancer prevention. Geneva: World Health Organisation; 2013.

11. Mustafa RA, Santesso N, Khatib R, et al. Systematic reviews and meta-analyses of the accuracy of HPV tests, visual inspection with acetic acid, cytology, and colposcopy. Int J Gynecol Obstet [serial online]. 2016 Mar [cited 2019 Nov 28];132(3):259-65. Available from: https://doi.org/10.1016/j.ijgo.2015.07.024

12. Einstein MH, Smith KM, Davis TE, et al. Clinical evaluation of the cartridge-based GeneXpert human papillomavirus assay in women referred for colposcopy. J Clin Microbiol. 2014;52(6):2089-2095. https://doi.org/10.1128/JCM.00176-14

13. Qiao Y lin, Sellors JW, Eder PS, et al. A new HPV-DNA test for cervical-cancer screening in developing regions: A cross-sectional study of clinical accuracy in rural China. Lancet Oncol. 2008 Oct;9(10):929-936. https://doi.org/10.1016/ S1470-2045(08)70210-9

14. JSI. Lessons learned - HPV vaccine nationwide introduction in Zimbabwe. Harare: John Snow, Inc; 2018.

15. Fitzpatrick MB, Dube Mandishora RS, Katzenstein DA, et al. hrHPV prevalence and type distribution in rural Zimbabwe: A community-based self-collection study using near-point-of-care GeneXpert HPV testing. Int J Infect Dis. 2019 May 01;82:21-29. https://doi.org/10.1016/j.ijid.2019.02.022

16. Thistle P, Parpia R, Pain D, Lee H, Manasa J, Schnipper LE. Prevalence and subtype distribution of high-risk human papillomavirus among women presenting for cervical cancer screening at Karanda Mission Hospital. JCO Glob Oncol. 2020 Aug 12;6(6):1276-1281. https://doi.org/10.1200/go.20.00286 
17. Menon S, Wusiman A, Boily MC, et al. Epidemiology of HPV genotypes among HIV positive women in Kenya: A systematic review and meta-analysis. PLoS One. 2016;11(10):e0163965. https://doi.org/10.1371/journal.pone.0163965

18. Ezechi OC, Ostergren PO, Nwaokorie FO, Ujah IAO, Odberg Pettersson K. The burden, distribution and risk factors for cervical oncogenic human papilloma virus infection in HIV positive Nigerian women. Virol J. 2014 Jan 15;11:5. https://doi. org/10.1186/1743-422x-11-5

19. Reddy D, Njala J, Stocker P, et al. High-risk human papillomavirus in HIVinfected women undergoing cervical cancer screening in Lilongwe, Malawi: A pilot study. Int J STD AIDS. 2015;26(6):379-387. https://doi.org/10.1177/0956 462414539149

20. Firnhaber C, Van Le H, Pettifor A, et al. Association between cervical dysplasia and human papillomavirus in HIV seropositive women from Johannesburg South Africa. Cancer Causes Control. 2010 Mar 01;21(3):433-443. https://doi. org/10.1007/s10552-009-9475-z

21. Fukuchi E, Sawaya GF, Chirenje $M$, et al. Cervical human papillomavirus incidence and persistence in a cohort of HIV-negative women in Zimbabwe. Sex Transm Dis. 2009 May;36(5):305-311. https://doi.org/10.1097/OLQ.0b013e31 8194eb76

22. Macleod IJ, O'donnell B, Moyo S, et al. Prevalence of human papillomavirus genotypes and associated cervical squamous intraepithelial lesions in HIV infected women in Botswana. J Med Virol. 2011;83(10):1689-1695. https://doi. org/10.1002/jmv.22178

23. Shamu T, Chimbetete $C$, Shawarira-Bote $S$, Mudzviti T, Luthy R. Outcomes of an HIV cohort after a decade of comprehensive care at Newlands Clinic in Harare, Zimbabwe: TENART cohort. PLoS One [serial online]. 2017 Oct 24 [cited 2018 Dec 12];12(10):e0186726. Available from: https://doi.org/10.1371/journal.pone. 0186726

24. Akbari A, Vanden Broeck D, Benoy I, Padalko E, Bogers J, Arbyn M. Validation of intra- and inter-laboratory reproducibility of the Xpert HPV assay according to the international guidelines for cervical cancer screening. Virol J. 2018 Oct 29;15(1):166. https://doi.org/10.1186/s12985-018-1076-6

25. Ndizeye Z, Broeck DV, Lebelo RL, et al. Prevalence and genotype-specific distribution of human papillomavirus in Burundi according to HIV status and urban or rural residence and its implications for control. PLoS One [serial online]. 2019 [cited 2020 Mar 25];14(6):e0209303. Available from: https://doi. org/10.1371/journal.pone.0209303

26. Menon SS, Rossi R, Harebottle R, Mabeya H, Vanden Broeck D. Distribution of human papillomaviruses and bacterial vaginosis in HIV positive women with abnormal cytology in Mombasa, Kenya. Infect Agent Cancer. 2016;11:17. https:// doi.org/10.1186/s13027-016-0061-1

27. Clifford GM, Tully S, Franceschi S. Carcinogenicity of human papillomavirus (HPV) types in HIV-positive women: A meta-analysis from HPV infection to cervica cancer. Clin Infect Dis. 2017 May 01;64(9):1228-1235. https://doi.org/10.1093/ cid/cix135
28. Clifford GM, Gallus S, Herrero R, et al. Worldwide distribution of human papillomavirus types in cytologically normal women in the International Agency for Research on Cancer HPV prevalence surveys: A pooled analysis. Lancet. 2005 Sep 17;366(9490):991-998. https://doi.org/10.1016/s0140-6736(05)67069-9

29. Parham GP, Sahasrabuddhe VV, Mwanahamuntu MH, et al. Prevalence and predictors of squamous intraepithelial lesions of the cervix in HIV-infected women in Lusaka, Zambia. Gynecol Oncol. 2006;103(3):1017-1022. https://doi.org/ 10.1016/j.ygyno.2006.06.015

30. Akarolo-Anthony SN, Al-Mujtaba M, Famooto AO, et al. HIV associated high-risk HPV infection among Nigerian women. BMC Infect Dis. 2013 Nov 05;13:521. https://doi.org/10.1186/1471-2334-13-521

31. Demarco M, Hyun N, Carter-Pokras O, et al. A study of type-specific HPV natural history and implications for contemporary cervical cancer screening programs. EClinicalMedicine. 2020;22:100293. https://doi.org/10.1016/j.eclinm.2020.100293

32. amFAR, The Foundation for AIDS Research (November, 2016) Cervical cancer, Human papillomavirus (HPV), and HPV vaccines in Southeast Asia [homepage on the Internet]. 2016 [cited 2020 May 01]. Available from: www.amfar.org

33. Van Der Weele P, Breeuwsma M, Donken R, et al. Effect of the bivalent HPV vaccine on viral load of vaccine and non-vaccine HPV types in incident clearing and persistent infections in young Dutch females. PLoS One. 2019 Mar 01;14(3):e0212927. https://doi.org/10.1371/journal.pone.0212927

34. Richter K, Becker P, Horton A, Dreyer G. Age-specific prevalence of cervical human papillomavirus infection and cytological abnormalities in women in Gauteng Province. S Afr Med J [serial online]. 2013 Mar 01;103(5):313. https://doi. org/10.7196/SAMJ.6514

35. Van Aardt MC, Dreyer G, Richter KL, Becker P. Human papillomavirus-type distribution in South African women without cytological abnormalities: A periurban study. S Afr J Gynaecol Oncol [serial online]. 2013 Jan 15;5(Suppl 1): S21-S27. https://doi.org/10.1080/20742835.2013.11441218

36. Auwal I, Aminu M, Atanda A, Tukur J, Sarkinfada F. Prevalence and risk factors of high risk human papillomavirus infections among women attending gynaecology
clinics in Kano, Northern Nigeria. Bayero J Pure Appl Sci. 2014 Jan 07;6(1):67. https://doi.org/10.4314/bajopas.v6i1.14

37. Akarolo-Anthony SN, Famooto AO, Dareng EO, et al. Age-specific prevalence of human papilloma virus infection among Nigerian women. BMC Public Health. 2014 Jun 27;14:656. https://doi.org/10.1186/1471-2458-14-656

38. Menon S, Rossi R, Zdraveska N, et al. Associations between highly active antiretroviral therapy and the presence of HPV, premalignant and malignant cervical lesions in sub-Saharan Africa: A systematic review: Current evidence and directions for future research. BMJ Open. 2017 Aug 04:7(8):e015123. https://doi. org/10.1136/bmjopen-2016-015123

39. Schiffman M, Glass AG, Wentzensen N, et al. A long-term prospective study of type-specific human papillomavirus infection and risk of cervical neoplasia among 20,000 women in the Portland Kaiser cohort study. Cancer Epidemiol Biomarkers Prev. 2011;20(7):1398-1409. https://doi.org/10.1158/1055-9965.EPI-11-0206 